\title{
REPRESENTATION OF $H^{p}$-FUNCTIONS
}

\author{
BY D. J. PATIL
}

Communicated by R. C. Buck, February 7, 1972

\begin{abstract}
Let $E$ be a set of positive measure on the unit circle. Let $f \in H^{p}(1 \leqq p \leqq \infty)$ and $g$ be the restriction of $f$ to $E$. It is shown that functions $g_{\lambda}, \lambda>0$, can be constructed from $g$ so that $g_{\lambda} \rightarrow f$. We also characterize those functions $g$ on $E$ which are restrictions of functions in $H^{p}(1<p \leqq \infty)$.
\end{abstract}

In the following, the space $H^{p}(1 \leqq p \leqq \infty)$ will, according to the context, be either the Hardy class of analytic functions in the open unit disc $D$ or the space of the corresponding boundary value functions, viz the subspace of "analytic" functions in $L^{p}(C), C$ being the unit circle. If $E \subset C$ has positive measure then it is well known (see [3]) that a function in $H^{p}$ cannot vanish on $E$ without being identically zero. Thus, theoretically at least, $f \in H^{p}$ is uniquely "determined" by its values on $E$. In the present work we address ourselves to the problem of recovering functions in $H^{p}$ from their restrictions to $E$. Theorem I gives an explicit constructive solution to this problem. The allied problem of characterizing the restrictions to $E$ of functions in $H^{p}(1<p \leqq \infty)$ is solved in Theorem II. To the best of our knowledge, the only known results relating to these problems are due to the author [4] where the case $p=2$ is dealt with.

THEOREM I. Let $E \subset C$ with $m(E)>0$. Suppose that $1 \leqq p \leqq \infty, f \in H^{p}$ and that $g$ is the restriction of $f$ to $E$. For each $\lambda>0$ define analytic functions $h_{\lambda}, g_{\lambda}$ on $D$ by

$$
\begin{aligned}
& h_{\lambda}(z)=\exp \left\{-\frac{1}{4 \pi} \log (1+\lambda) \int_{E} \frac{e^{i \theta}+z}{e^{i \theta}-z} d \theta\right\}, \quad z \in D, \\
& g_{\lambda}(z)=\lambda h_{\lambda}(z) \frac{1}{2 \pi i} \int_{E} \frac{\bar{h}_{\lambda}(w) g(w) d w}{w-z}, \quad z \in D .
\end{aligned}
$$

Then as $\lambda \rightarrow \infty, g_{\lambda} \rightarrow f$ uniformly on compact subsets of $D$. Moreover for $1<p<\infty$ we also have $\left\|g_{\lambda}-f\right\|_{p} \rightarrow 0$ as $\lambda \rightarrow \infty$.

TheOREM II. Let $E \subset C$ with $0<m(E)<m(C)$. For $g \in L^{1}(E)$ let $g_{\lambda}$ be as in Theorem I. (a) If $1<p<\infty$ then a function $g \in L^{p}(E)$ is the restriction to $E$ of some $f \in H^{p}$ if and only if $\sup _{\lambda}>0\left\|g_{\lambda}\right\|_{p}<\infty$. (b) A function $g \in L^{\infty}(E)$ is the restriction to $E$ of some $f \in H^{\infty}$ if and only if $\sup _{p>1} \lim \sup _{\lambda \rightarrow \infty}\left\|g_{\lambda}\right\|_{p}$ $<\infty$.

AMS 1970 subject classifications. Primary 30A78, 30A88; Secondary 46E20, 30A86, 47B35. 
The proof of Theorem I will be based on a series of lemmas. First we recall some elementary properties of Toeplitz operators on $H^{p}$ spaces (for details in the special case $p=2$ see [1], and for the general case $1<p<\infty$ see [5]). Let $1<p<\infty$. For each $\varphi \in L^{\infty}$, the Toeplitz operator $T_{\varphi}$ is defined by $T_{\varphi} f=P(\varphi f), f \in H^{p}$, where $P$ is the natural projection of $L^{p}$ onto $H^{p}$. We need the following facts: (i) $\left\|T_{\varphi}\right\| \leqq C_{p}\|\varphi\|_{\infty}$, (ii) if $\varphi, \psi \in L^{\infty}$ and if either $\bar{\varphi} \in H^{\infty}$ or $\psi \in H^{\infty}$, then $T_{\varphi \psi}=T_{\varphi} T_{\psi}$. This latter fact immediately yields

Lemma 1. If $h, 1 / h \in H^{\infty}$ and $\varphi=|h|^{-2}$, then the Toeplitz operator $T_{\varphi}$ is invertible and $T_{\varphi}^{-1}=T_{h} T_{\bar{h}}$.

Proof. $T_{h} T_{\bar{h}} T_{\varphi}=T_{h}\left(T_{\bar{h}} T_{1 / \bar{h}}\right) T_{1 / h}=T_{h} T_{1 / h}=I$, etc.

Let $\chi_{E}$ be the characteristic function of the set $E$ and let for $\lambda>0$, $\varphi_{\lambda}=1+\lambda \chi_{E}$. Then the function $h_{\lambda}$ defined in Theorem I satisfies, $1 / \varphi_{\lambda}=h_{\lambda} h_{\lambda}$. Also $h_{\lambda}, 1 / h_{\lambda} \in H^{\infty}$. Thus by Lemma 1 , we have

LEMMA 2. $T_{\varphi_{\lambda}}$ is invertible and $T_{\varphi_{\lambda}}^{-1}=T_{h_{\lambda}} T_{\bar{h}_{\lambda}}$.

Lemma 3. Define for each $a \in D, e_{a}(z)=1 /(1-\bar{a} z), z \in D$. Then $e_{a} \in H^{p}$, $1 \leqq p \leqq \infty$, and if $T_{\varphi_{2}}$ is treated as an operator on $H^{p}(1<p<\infty)$, we have $T_{\varphi_{\lambda}}^{-1} e_{a}=h_{\lambda}(a) h_{\lambda} e_{a}$.

Proof. For each $g \in H^{q}(q=p /(p-1))$, we have $\left(T_{h_{2}} e_{a}, g\right)=\left(e_{a}, h_{\lambda} g\right)$ $=h_{\lambda}(a) \bar{g}(a)=h_{\lambda}(a)\left(e_{a}, g\right)$. Thus $T_{\bar{h}_{2}} e_{a}=h_{\lambda}(a) e_{a}$. An appeal to Lemma 2 finishes the proof.

Lemma 4. Let $K$ be a compact subset of $D$ and $1 \leqq p \leqq \infty$. Then as $\lambda \rightarrow \infty,\left\|h_{\lambda}(a) h_{\lambda} e_{a}\right\|_{p} \rightarrow 0$ uniformly for $a \in K$.

Proof. We note that ||$h_{\lambda} \|_{\infty} \leqq 1$ and $\left|h_{\lambda}(a)\right| \leqq(1+\lambda)^{-\alpha}$ where $\alpha>0$ and $\alpha$ depends on $|a|$.

Let now $S$ be the Toeplitz operator on $H^{p}(1<p<\infty)$ corresponding to the characteristic function $\chi_{E}$ of $E$. Then since $I+\lambda S=T_{\varphi},(I+\lambda S)^{-1}$ exists by Lemma 2 . Also by Lemma $4,\left\|(I+\lambda S)^{-1} e_{a}\right\|_{p} \rightarrow 0$ as $\lambda \rightarrow \infty$. By Lemma 2 and fact (i) about Toeplitz operators we also have

$$
\left\|(I+\lambda S)^{-1}\right\|=\left\|T_{h_{\lambda}} T_{\bar{h}_{\lambda}}\right\| \leqq\left\|h_{\lambda}\right\|_{\infty}^{2} C_{p}^{2} \leqq C_{p}^{2} .
$$

Noting that $\left\{e_{a}: a \in D\right\}$ is a fundamental set in $H^{p}$, we therefore obtain (cf., e.g., $\left[3\right.$, p. 55]) that $\left\|(I+\lambda S)^{-1} f\right\|_{p} \rightarrow 0$ for every $f \in H^{p}$. Noting that for $f \in H^{p},(I+\lambda S)^{-1} f=f-\lambda(I+\lambda S)^{-1} S f$, we get

Lemma 5. If $1<p<\infty$ and $f \in H^{p}$, then as $\lambda \rightarrow \infty$,

$$
\left\|\lambda(I+\lambda S)^{-1} S f-f\right\|_{p} \rightarrow 0 \text {. }
$$


The proof of Theorem I (for $1<p<\infty$ ) will be complete if we show that $g_{\lambda}=\lambda(I+\lambda S)^{-1} S f$. But this is routine: For $z \in D$,

$$
\begin{aligned}
\left(\lambda(I+\lambda S)^{-1} S f, e_{z}\right) & =\lambda\left(S f,(I+\lambda S)^{-1} e_{z}\right)=\lambda\left(\chi_{E} f,(I+\lambda S)^{-1} e_{z}\right) \\
& =\lambda\left(f,(I+\lambda S)^{-1} e_{z}\right)_{E}=\lambda\left(f, \bar{h}_{\lambda}(z) h_{\lambda} e_{z}\right)_{E} .
\end{aligned}
$$

In the above chain of equalities, the first is a consequence of the fact that $(I+\lambda S)^{*}$ is the operator $(I+\lambda S)$ on $H^{q}(q=p /(p-1))$ and the last results from Lemma 3. The notation (, $)_{E}$ denotes the "inner product" over the set $E$. Now it can be readily checked that $\lambda\left(f, h_{\lambda}(z) h_{\lambda} e_{z}\right)_{E}$ is the same as the defining expression for $g_{\lambda}(z)$.

The case $p=\infty$ is easy. If $f \in H^{\infty}$ then since $f$ is also in $H^{2}$, by the preceding, $\left\|g_{\lambda}-f\right\|_{2} \rightarrow 0$ and hence $g_{\lambda} \rightarrow f$ uniformly on compact subsets of $D$.

Turning to the case $p=1$, let $f \in H^{1}$. For $0<r<1$, define $f_{r}$ by $f_{r}\left(e^{i \theta}\right)$ $=f\left(r e^{i \theta}\right)$. Then as is well known, $\left\|f_{r}\right\|_{1} \leqq\|f\|_{1}$ and $\left\|f_{r}-f\right\|_{1} \rightarrow 0$ as $r \rightarrow 1$. Let us define, for each $\lambda>0, f_{r, \lambda}$ by

$$
f_{r, \lambda}(z)=\lambda h_{\lambda}(z) \frac{1}{2 \pi i} \int_{E} \frac{h_{\lambda}(w) f_{r}(w)}{w-z} d w, \quad z \in D .
$$

Then we see that, for every compact set $K \subset D$, the following statements hold uniformly in $K:(1) f_{r, \lambda} \rightarrow g_{\lambda}$ as $r \rightarrow 1$, (2) $f_{r} \rightarrow f$ as $r \rightarrow 1$, (3) $f_{r, \lambda} \rightarrow f_{r}$ as $\lambda \rightarrow \infty$. The less trivial of these statements, viz. (3), follows because $f_{r} \in H^{2}$ and the case $p=2$ of the theorem applies. If we show further that the convergence in $(3)$ is also uniform for $r$ in $(0,1)$ then we can conclude that $g_{\lambda} \rightarrow f$ as $\lambda \rightarrow \infty$ uniformly in $K$ and the proof of the theorem for $p=1$ will be complete. For this purpose, remembering that $f \in H^{2}$ we have for each $z \in K$,

$$
\begin{aligned}
f_{r, \lambda}(z)-f_{r}(z) & =\left(\lambda(I+\lambda S)^{-1} S f_{r}-f_{r}, e_{z}\right)=\left((I+\lambda S)^{-1} f_{r}, e_{z}\right) \\
& =\left(f_{r},(I+\lambda S)^{-1} e_{z}\right)=\left(f_{r}, h_{\lambda}(z) h_{\lambda} e_{z}\right) .
\end{aligned}
$$

Hence we obtain

$$
\left|f_{r}(z)-f_{r, \lambda}(z)\right| \leqq\left\|f_{r}\right\|_{1}\left\|\bar{h}_{\lambda}(z) h_{\lambda} e_{z}\right\|_{\infty} \leqq\|f\|_{1}\left\|\bar{h}_{\lambda}(z) h_{\lambda} e_{z}\right\|_{\infty} .
$$

The last term is independent of $r$ and Lemma $4(p=\infty)$ does the job.

Proof of TheOREM II. The "only if" parts are evident from Theorem I. As for the "if" part in (a), the boundedness of $\left\{\left\|g_{\lambda}\right\|_{p}\right\}$ together with the weak* compactness of closed balls in $H^{p}$ provide us with a sequence $\lambda_{n} \rightarrow \infty$ such that $g_{\lambda_{n}}$ converges weak* to some $f$ in $H^{p}$. Let $g_{1} \in L^{p}(C)$ be defined by setting $g_{1}=g$ on $E$ and $g_{1}=0$ otherwise. Denote $P g_{1}$ by $\tilde{g}$. From the discussion following Lemma 5 , it can be seen that

$$
g_{\lambda}=\lambda(I+\lambda S)^{-1} \tilde{g} \text {. }
$$


Thus for every $k \in H^{q}(q=p /(p-1)),\left(\lambda_{n}\left(I+\lambda_{n} S\right)^{-1} S \tilde{g}, k\right)=\left(g_{\lambda_{n}}, S k\right)$ $\rightarrow(f, S k)=(S f, k)$, while by Lemma 5 , the first of these inner products converges to $(\hat{g}, k)$. Hence $\hat{g}=S f$. This means that the Fourier coefficients $\left(\left(f-g_{1}\right) \chi_{E}\right)^{-}(n)$ are zero for $n \geqq 0$. In other words, $\left(f-\bar{g}_{1}\right) \chi_{E} \in H^{p}$. Since $m(C \backslash E)>0$, we must have $f=g_{1}$ on $E$.

For proving the "if" part in (b) we need to make just two observations. First, $g \in L^{\infty}(E)$ implies $g_{\lambda} \in H^{p}$ for each $p<\infty$ and hence part (a) gives $f$ belonging to $H^{p}$ for all $p<\infty$ and such that $g$ is the restriction to $E$ of $f$. Secondly, $\left\|g_{\lambda}\right\|_{p} \rightarrow\|f\|_{p}$ as $\lambda \rightarrow \infty$ and $\|f\|_{p} \rightarrow\|f\|_{\infty}$ as $p \rightarrow \infty$. The details are left to the reader.

Remarks. 1. In the proof of Theorem I, we did not use the F. \& M. Riesz Theorem. We thus obtain a new proof of the statement: if $f \in H^{p}$ $(1 \leqq p \leqq \infty), f=0$ on $E, m(E)>0$, then $f=0$.

2. Theorem I points out a way which enables us to draw conclusions about the properties of a holomorphic function from the knowledge of its values on an arc. It is possible to obtain results parallel to the classical Cauchy theory where we now have integrals over a curve which may not be closed. Details of these and other related results will be published elsewhere.

\section{REFERENCES}

1. A. Brown and P. R. Halmos, Algebraic properties of Toeplitz operators, J. Reine Angew. Math. $213(1963 / 64), 89-102$. MR 28 \#3350; 30, 1205.

2. N. Dunford and J. T. Schwartz, Linear operators. I: General theory, Pure and Appl. Math., vol. 7, Interscience, New York, 1958. MR 22 \#302.

3. P. L. Duren, Theory of $H^{p}$ spaces, Academic Press, New York, 1970.

4. D. J. Patil, Recapturing $\boldsymbol{H}^{2}$-functions from boundary values on small sets (unpublished).

5. H. Widom, Toeplitz operators on $H_{p}$, Pacific J. Math. 19 (1966), 573-582. MR 34 \# 1859.

Department of Mathematics, University of Wisconsin, Mil WaUKee, Wisconsin 53201 